\title{
Phenotypic markers of oral keratinocytes seeded on two distinct 3D oral mucosa models
}

\author{
Fernanda G. Basso $^{\mathrm{a}, *}$, Taisa N. Pansani ${ }^{\mathrm{a}}$, Cyntia L. Marcelo ${ }^{\mathrm{b}}$, Carlos Alberto de Souza Costa ${ }^{\mathrm{a}}$, \\ Josimeri Hebling ${ }^{\mathrm{a}}$, Stephen E. Feinberg ${ }^{\mathrm{b}}$

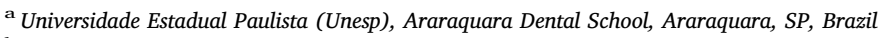 \\ ${ }^{\mathrm{b}}$ University of Michighan, Ann Arbor, MI, United States
}

\section{A R T I C L E I N F O}

\section{Keywords:}

Cell culture techniques

Gingival fibroblasts

Organotypic cell culture

\begin{abstract}
A B S T R A C T
This study validates the use of a full-thickness oral mucosa model for in vitro studies with a collagen type I matrix, by comparison of this model with two other 3D oral mucosa models: human-sourced and porcine acellular dermal matrices (AlloDerm ${ }^{\circledR} /$ Strattice $^{\circledast}$, respectively). For the collagen matrix model, gingival fibroblasts were seeded either onto the dermal side of the AlloDerm ${ }^{\circledast}$ and Strattice ${ }^{\circledast}$ matrices or within the collagen matrices in complete culture medium (DMEM). For all scaffolds, DMEM was replaced every $24 \mathrm{~h}$ up to $72 \mathrm{~h}$. For the full-thickness oral mucosa models, $72 \mathrm{~h}$ after fibroblast seeding, oral keratinocytes were seeded on the epidermal sides of AlloDerm ${ }^{\circledR}$ and Strattice ${ }^{\circledR}$ matrices or collagen matrices. All matrices and models were subjected to histological analysis, complementing phenotypic characterization by evaluation of glucose consumption, cell proliferation, gene expression and synthesis of growth factors. A higher fibroblast ratio was observed for the collagen matrix, in which the distribution of gingival fibroblasts was also more homogeneous. Metabolism, proliferation, and gene expression and synthesis of VEGF of these cells were also increased for the collagen matrix. All matrices provided a suitable substrate for oral keratinocytes adhesion, proliferation, and phenotypic expression; however, higher proliferation, stratification, and differentiation were noted when oral keratinocytes were seeded on the dermal matrices.
\end{abstract}

\section{Introduction}

Monolayer cell culture has been the model used extensively for assessing the responses and interactions of different cell lines subjected to therapies (Moharamzadeh et al., 2007, 2012). In addition to the significant advances obtained with this model, it has shown limited simulation of cell interactions and phenotypes, since cell culture confluence may interfere with the morphology and behavior of cells and significantly reduce some cell functions, such as migration and proliferation (Moharamzadeh et al., 2007, 2012).

To improve laboratory studies of mammalian cells, three-dimensional (3D) cell culture models were developed. These models allow for interactions among cells of the same and different tissues as well as with the extracellular matrix, mimicking in vivo conditions (Feinberg et al., 2005; Moharamzadeh et al., 2007).

Several 3D oral mucosa models have been proposed involving different techniques and materials (Donetti et al., 2009; Klausner et al., 2007; Lambros et al., 2011; Moharamzadeh et al., 2007; Tobita et al., 2010). The acellular human dermal matrix (AlloDerm ${ }^{\oplus}$, LifeCell,
Bridgewater, NJ, USA) is a standardized and easy-to-handle material extensively used for the development of organotypic cell culture of oral mucosa and skin (Feinberg et al., 2005; Izumi et al., 1999, 2004; Tobita et al., 2010). Similarly, a porcine acellular dermal matrix has also been applied to produce a full 3D epithelium (Basso et al., 2016a). Collagen scaffolds have also been widely used to create 3D cell culture (Moharamzadeh et al., 2012).

The majority of 3D oral mucosa models have focused on the achievement of a morphological equivalent of epithelial tissue (Moharamzadeh et al., 2012). However, it has already been demonstrated that the morphology and phenotypes of epithelial cells are directly related to the cross-communication of these cells to the underlying mesenchymal tissue, mainly represented by fibroblasts and collagen fibers (Hakkinen et al., 2000; Werner et al., 2007). Therefore, the application of organotypic models of oral mucosa containing epidermal and mesenchymal components for in vitro studies could provide more significant results regarding physiological and pathological conditions.

Unfortunately, only scarce data are available regarding specific

\footnotetext{
* Corresponding author at: Universidade Estadual Paulista (Unesp), Araraquara Dental School, R. Humaita, 1680, 14801-375 Araraquara, SP, Brasil.

E-mail address: fergbasso@gmail.com (F.G. Basso).
} 
techniques and materials for a 3D gingival fibroblast cell culture capable of better mimicking cellular gingival connective tissue in vivo (Rakhorst et al., 2006; Colley et al., 2011; Dongari-Bagtzoglou and Kashleva, 2006; Moharamzadeh et al., 2012; Rodrigues et al., 2010). Therefore, this investigation provides a comparative analysis of two acellular dermal matrices and collagen matrices for the development of a morphologically and phenotypically feasible full 3D cell culture and a comparison of both matrices for the production of a model that can simulate gingival connective tissue and a full-thickness oral mucosa model for further laboratory studies.

\section{Materials and methods}

\subsection{Cell isolation and culture - Oral keratinocytes and gingival fibroblasts}

Gingival fibroblasts (GF) and oral keratinocytes (OK) were isolated from the gingival tissue of a healthy young patient, following the procedures approved by the Ethics Committee of the Araraquara School of Dentistry (CAAE: 14342113.7.0000.5416). Oral keratinocytes were isolated by enzymatic digestion with $0.04 \%$ trypsin (Gibco, Carlsbad, CA, USA) for $8 \mathrm{~h}$ at room temperature and were maintained in EpiLife cell culture medium (Gibco), supplemented with epidermal growth factor and calcium chloride $(0.06 \mathrm{mM})$. Gingival fibroblasts were also obtained by enzymatic digestion with collagenase type I (Worthington Biochemical Corp., Lakewood, NJ, USA) and were maintained in Dulbecco's Modified Eagle's Medium (DMEM - Gibco) containing gentamycin, fungizone (Gibco), and $10 \%$ fetal bovine serum (FBS). Subculture processes were performed with $0.25 \%$ Trypsin solution (Gibco).

\section{2. $3 D$ cell culture models}

To provide a full comparison between dermal and collagenous matrices, two distinct 3D cell culture models were proposed for the study. The first model was obtained by the seeding of only gingival fibroblasts, providing a cellular dermal matrix; for the second model, oral keratinocytes were seeded in addition to gingival fibroblasts, providing a full-thickness oral mucosa model, containing a cellular dermal matrix and epithelial component.

\subsection{Cell seeding in dermal matrices}

For the dermal matrices model, human-sourced (AlloDerm ${ }^{\circledR}$, LifeCell Corp., Branchburg, NJ, USA) and porcine-sourced (Strattice ${ }^{\circledR}$, LifeCell Corporation) matrices were selected. Before cells were seeded, the AlloDerm ${ }^{\circledast}$ or Strattice ${ }^{\circledR}$ matrix was cut into rounded samples $0.6 \mathrm{~cm}$ in diameter by means of a sterilized scalpel $(n=4)$. Matrices were hydrated with phosphate-buffered saline solution (PBS) for $45 \mathrm{~min}(3 \times$ for $15 \mathrm{~min}$ each).

For the first model, where only gingival fibroblasts were seeded (GF), after hydration with PBS, these matrices were placed at the bottoms of 48-well plates with the dermal side facing up, and cells were seeded on them $\left(1 \times 10^{5}\right.$ cells/well) in DMEM culture medium (Gibco, Carlsbad, CA, USA), supplemented with $10 \%$ fetal bovine serum (FBS) (Gibco). Then, the well-plates containing matrices and cells were maintained in an incubator for $72 \mathrm{~h}$ at $37^{\circ} \mathrm{C}$ and $5 \%$ of $\mathrm{CO}_{2}$. The culture medium was replaced every $24 \mathrm{~h}$.

For the full-thickness oral mucosa model (OK/GF), after hydration with PBS, dermal matrices were coated with collagen type IV $(1 \mathrm{mg} / \mathrm{mL}$ - Sigma-Aldrich, St. Louis, MO, USA) and incubated overnight at $4{ }^{\circ} \mathrm{C}$. Then, after fibroblasts seeding according to previously described, matrices were transferred to another well with the epidermal side facing up, and oral keratinocytes were seeded $\left(2 \times 10^{5}\right.$ cells/matrix $)$ in EpiLife culture medium (Gibco) containing $6 \mathrm{mM}$ of calcium chloride. Matrices were kept submerged for 4 days and then transferred to an airliquid interface with transwell inserts and maintained for an additional
7 days, with culture medium replacement every $48 \mathrm{~h}$ (Basso et al., 2016a).

\subsection{Cell seeding in collagen matrix}

The three-dimensional cell culture model with rat collagen matrix was developed following an adaptation of the model previously described by Dongari-Bagtzoglou and Kashleva (2006), as already demonstrated in Basso et al. (2016b). First, an acellular matrix was obtained by mixing cold DMEM supplemented with $10 \%$ of FBS (Gibco) with rat collagen type I (BD Biosciences, Franklin Lakes, NJ, USA), to a final concentration of $0.77 \mathrm{mg} / \mathrm{mL}$. A $200-\mu \mathrm{L}$ quantity of this matrix was added to each well of a 24-well plate and incubated for $30 \mathrm{~min}$ at room temperature.

For the gingival fibroblast model, after the acellular matrix was achieved, these cells were trypsinized and added $\left(10^{5}\right.$ cells $\left./ \mathrm{mL}\right)$ to a mixture of DMEM and collagen at $0.77 \mathrm{mg} / \mathrm{mL}(n=4)$. This solution was applied to the acellular collagen ( $1 \mathrm{~mL}$ per well), which was maintained for $1 \mathrm{~h}$ at room temperature followed by incubation for an additional $\mathrm{h}$ at $37^{\circ} \mathrm{C}$ and $5 \%$ of $\mathrm{CO}_{2}$. Then, $1 \mathrm{~mL}$ of DMEM was added to each well, and samples were incubated for $72 \mathrm{~h}$. The culture medium was replaced every $24 \mathrm{~h}$ (Basso et al., 2016b).

After 3 days, the collagen matrices exhibited notable contraction, due to the stabilization of collagen, such that their morphology was similar to that of the dermal matrices (approximately $0.6 \mathrm{~cm}$ in diameter).

For the full-thickness oral mucosa model, after $72 \mathrm{~h}$, matrices were transferred to transwell inserts, and oral keratinocytes were seeded on the top of each collagen matrix. Then, the matrices were kept submerged for 4 days and then transferred to an air-liquid interface for an additional 7 days, with medium replacement every $48 \mathrm{~h}$.

\subsection{Histological evaluation}

Samples were fixed in $10 \%$ formalin at room temperature and then subjected to the standard histological process of washing and dehydration, and then to paraffin infiltration. Serial sections ( $5 \mu \mathrm{m}$ thick) were obtained in a rotating microtome (RM2125RT, Leica Microsystems, Wetzlar, Germany) and stained with hematoxylin and eosin. Samples were analyzed in a photomicroscope (Olympus BX51, Olympus America Inc., Center Valley, PA, USA) for the morphology and distribution of oral fibroblasts seeded on the dermal and collagen matrices. The histological features determined in the 3D cell culture models were compared with those observed in sections obtained from normal gingiva donated by a healthy young patient.

\subsection{Glucose consumption}

Glucose consumption was detected for the determination of cell viability. This analysis was performed using culture supernatant that remained in contact with cells in the different models, during all procedures, by an end-point colorimetric assay (Glicose Liquiform, Labtest Diagnóstica SA, Lagoa Santa, MG, Brazil), based on the reduction of 4aminoantipirin by glucose oxidase. Briefly, samples were incubated with glucose oxidase at $37^{\circ} \mathrm{C}$ and glucose concentration was determined by absorbance at $505 \mathrm{~nm}$ (Synergy H1).

As a negative control, glucose concentration of fresh culture medium was quantified. Then, glucose consumption was determined by means of comparison between fresh culture medium and each sample. Then, data was represented in percentage.

\subsection{Cell proliferation}

Cell proliferation was evaluated by the alamarBlue ${ }^{\circledR}$ assay. After $72 \mathrm{~h}$ for the gingival fibroblast model and after 11 days for the oral keratinocyte and full-thickness oral mucosa models, matrices were 

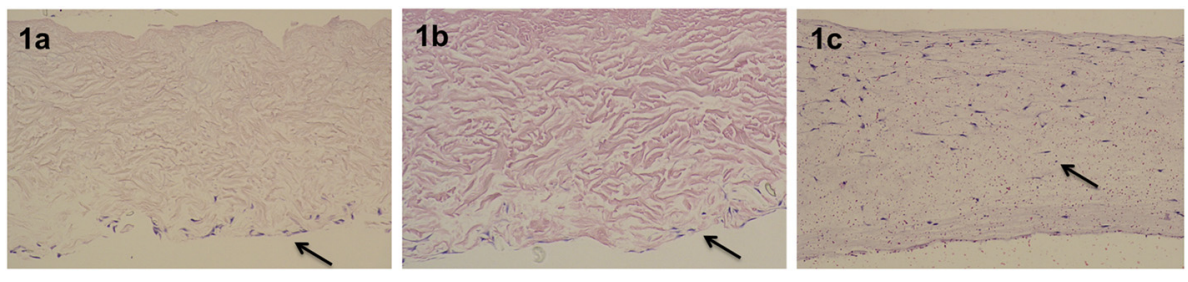

Fig. 1. Photomicrographs of 3D cell culture obtained from gingival fibroblasts - indicated by arrows seeded on (a) the AlloDerm ${ }^{\circledR}$ matrix, (b) the Strattice ${ }^{\circledR}$ matrix, or $(c)$ within collagen matrix $(\times 100)$.
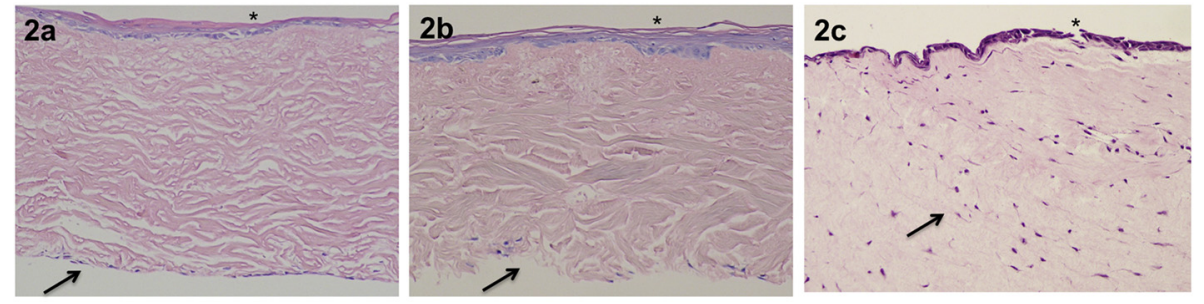

Fig. 2. Photomicrographs of 3D cell culture obtained from gingival fibroblasts - indicated by arrows - and oral keratinocytes - indicated by asterisks (*), seeded on (a) the AlloDerm ${ }^{\circledast}$ matrix, (b) the Strattice ${ }^{\circledast}$ matrix, or (c) within collagen matrix $(\times 100)$.

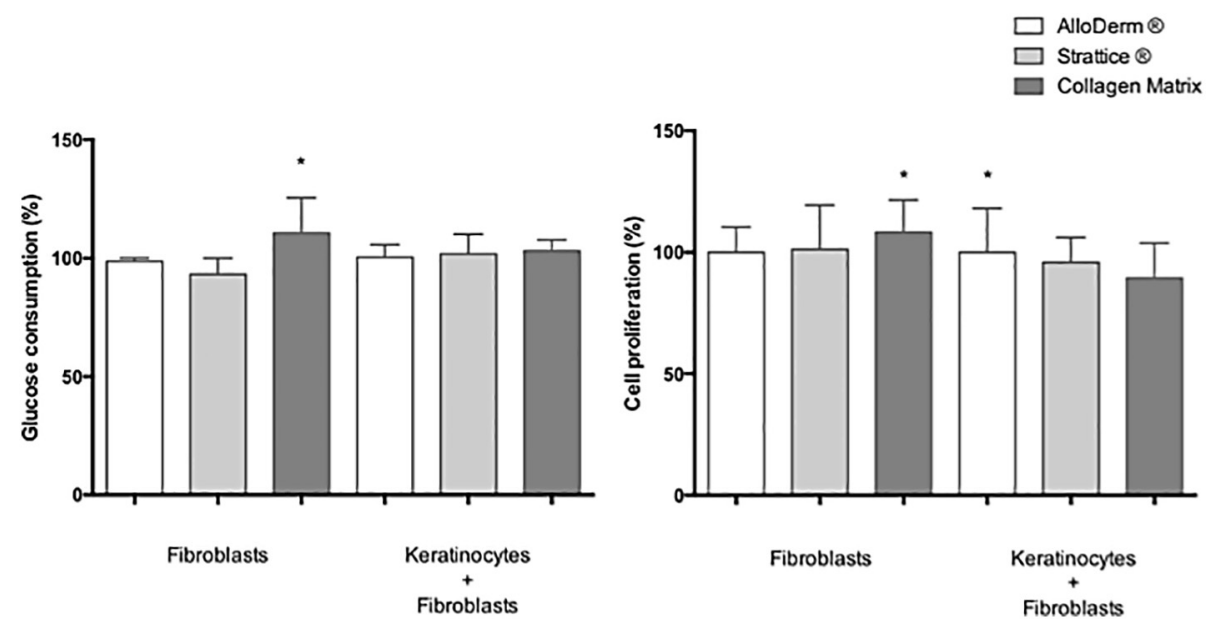

Fig. 3. Glucose consumption and cell proliferation of cells seeded on distinct cell culture models. Bars represent mean values \pm standard deviation; * indicates statistically significant difference among groups, based on cell lines applied (Tukey, $p<.05$ ).

transferred to a 24-well cell culture plate, and a solution containing $10 \%$ of alamarBlue ${ }^{\circledast}$ reagent in serum-free culture medium was applied to each sample. After a 4-hour incubation at $37^{\circ} \mathrm{C}$ and $5 \%$ of $\mathrm{CO}_{2}$, a $200-\mu \mathrm{L}$ aliquot of each sample was transferred to a 96 -well plate, and the fluorescence intensity was determined as the cell rate for each sample, by fluorimetry (Synergy - H1, BioTek, Winooski, VT, USA) (530 nm/590 nm excitation/emission) (Basso et al., 2016b).

\subsection{Gene expression}

The influence of different 3D cell culture models on the phenotypic characteristics of oral keratinocytes and gingival fibroblasts was demonstrated by evaluation of the gene expression of different growth factors. For the collagen matrix model, the expression of collagen type I (hCOL-I) and vascular endothelial growth factor (hVEGF) was evaluated, while for the full-thickness oral mucosa models, the expression of epidermal growth factor (hEGF) and keratinocyte growth factor (hKGF) was determined.

Gene expression was determined by quantitative PCR (qPCR). Total RNA of each sample was isolated by filtration, with the RNAqueous micro kit (Ambion, Grand Island, NY, USA). The High Capacity cDNA Reverse Transcriptase Kit (Applied Biosystems, Grand Island, NY, USA) was applied to provide cDNA synthesis, with $1 \mu \mathrm{g}$ of RNA for each sample and following the manufacturer's instructions. After reactions were prepared, samples were subjected to thermal cycling (Thermocycler, Bio-Rad, Hercules, CA, USA). qPCR reactions were prepared with Taqman Master Mix and Taqman Assays (Applied Biosystems) for experimental primers and endogenous control (betaActin). These reactions were performed in the Step One Plus Thermocycler and were analyzed by Step One Software.

mRNA concentrations for each primer and sample were normalized according to the results of endogenous control and were represented as fold-change. For GF/OK samples, gene expression represents mRNA for both cell lines, since it was not possible to isolate each genetic material.

\subsection{Protein synthesis}

Synthesis of growth factors was confirmed by the enzyme-linked immunoassay (ELISA), using specific kits for each growth factor, according to manufacture's recommendation (R\&D Systems).

\subsection{Statistical analysis}

Data for glucose consumption, cell proliferation, and gene expression showed normal distribution (Shapiro-Wilk, $\alpha=0.05$ ); therefore, parametric ANOVA and Tukey tests were applied for statistical analysis. All inferences were performed with $5 \%$ considered significant. 

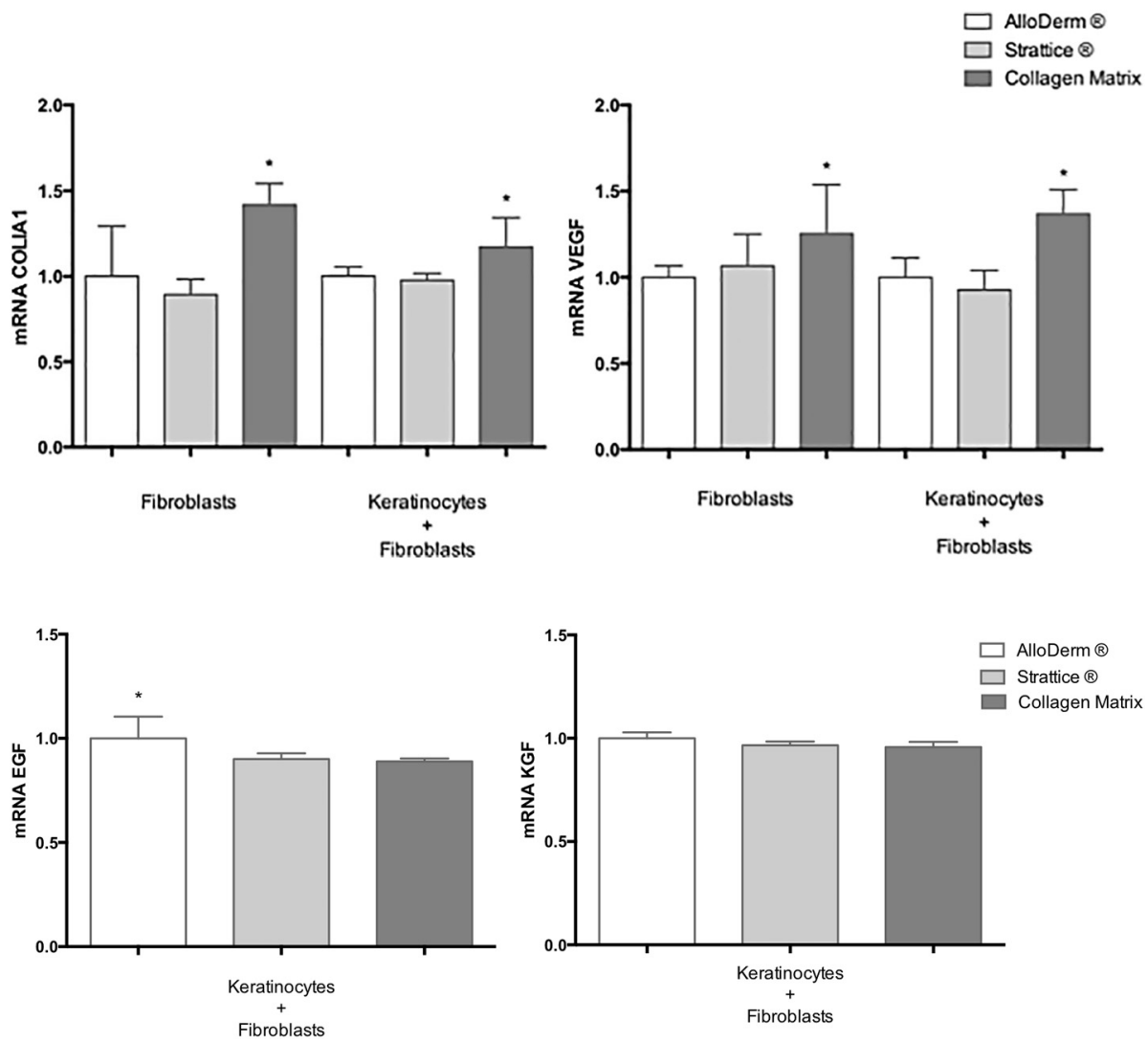

Fig. 4. Gene expression of hCOLIA1 and hVEGF by cells seeded on distinct cell culture models. Bars represent mean values \pm standard deviation; * indicates statistically significant difference among groups, based on cell lines applied (Tukey, $p<.05$ ). For GF/OK models, isolated mRNA is represents genetic material of both cell lines.
Fig. 5. Gene expression of hEGF and hKGF by oral keratinocytes seeded on distinct cell culture models. Bars represent mean values \pm standard deviation; * indicates statistically significant difference among groups, based on cell lines applied (Tukey, $p<.05$ ). For GF/OK models, isolated mRNA is represents genetic material of both cell lines.

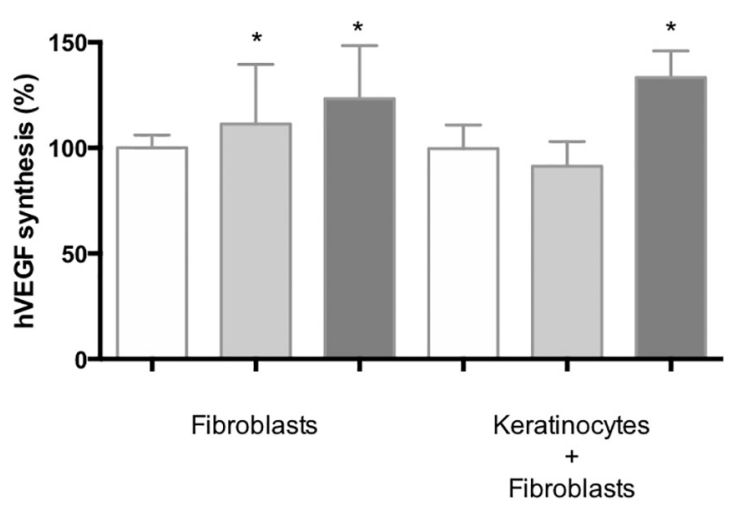

Fig. 6. Synthesis of hVEGF by cells seeded on distinct cell culture models. Bars represent mean values \pm standard deviation; * indicates statistically significant difference among groups, based on cell lines applied (Tukey, $p<.05$ ). For GF/OK models, samples contained proteins synthetized both cell lines.

\section{Results and discussion}

In spite of the numerous studies that have demonstrated the feasibility of different 3D models for epidermal tissue (Basso et al., 2016a; Donetti et al., 2009; Feinberg et al., 2005; Klausner et al., 2007; Moharamzadeh et al., 2007), there is still a lack of studies comparing these models in terms of the phenotypic characteristics of these cells. Therefore, some investigations aimed to demonstrate the development of 3D cell culture models using oral mucosal cells, which produced mainly organotypic models for oral epithelium (Basso et al., 2016a;
Lambros et al., 2011; Tobita et al., 2010). In contrast, few studies have provided an evaluation of the suitability of full-thickness oral mucosa models containing an underlying connective component presenting mesenchymal cells, mainly fibroblasts (Colley et al., 2011; DongariBagtzoglou and Kashleva, 2006; Moharamzadeh et al., 2012; Rodrigues et al., 2010). The homeostasis and repair of oral mucosa rely on the interaction of both mesenchymal and epidermal cells (Hakkinen et al., 2000; Werner et al., 2007). Therefore, the reproduction of this interaction in the development of three-dimensional cell models for in vitro studies must be considered.

Three-dimensional models obtained with AlloDerm ${ }^{\circledR}$ and Strattice ${ }^{\circledast}$ matrices demonstrated that fibroblasts were able to adhere to the dermal surface; however, in spite of this adhesion, a few cells showed migratory behavior, going further into the dermal matrices, while most fibroblasts exhibited poor capacity for migration within the collagen fibrils, remaining on the superficial layer of the dermal side of the product (Fig. $1 \mathrm{a}$ and $\mathrm{b}$ ).

Previous studies also demonstrated similar results, with sparse cell distribution and migration, suggesting that acellular dermal matrices are not the best model to represent gingival connective tissue (Rodrigues et al., 2010). The association of both cell strains, using this model for both dermal matrices, did not seem to present a significant influence for the oral mucosa cells, since epithelial cells showed morphology similar to that observed for the previous 3D model (Fig. 2a and b).

Rakhorst et al. (2006) applied a centrifugation method to improve insertion and distribution of fibroblasts within dermal matrices. By means of this method, cells showed a more uniform distribution, demonstrating that these matrices can be feasible for fibroblasts seeding when this technique is applied.

The lack of migration of fibroblasts within the dermal matrices can be related to the density of the collagen fibers of this scaffold. The morphological analysis of both dermal matrices demonstrates the compact distribution of these fibers, with few inter-fibrillary spaces 

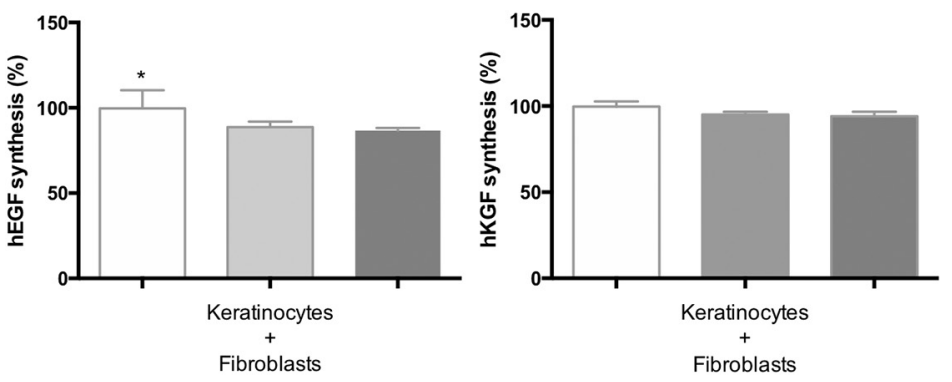

Fig. 7. Synthesis of hEGF and hKGF by oral keratinocytes seeded on distinct cell culture models. Bars represent mean values \pm standard deviation; * indicates statistically significant difference among groups, based on cell lines applied (Tukey, $p<.05$ ). For GF/OK models, isolated mRNA is represents genetic material of both cell lines.
(Figs. 1a,b and 2a,b).

For the experimental collagen matrix, gingival fibroblasts presented homogeneous distribution and morphology throughout the $3 \mathrm{D}$ culture model. These cells exhibited cytoplasmic projections similar to those observed in the sections obtained from normal gingival tissue (Fig. 1c). However, when the model was intended to obtain a full-thickness oral mucosa, lower adhesion and differentiation of oral keratinocytes were observed when compared to dermal matrices (Fig. 2c).

AlloDerm ${ }^{\circledR}$ matrices, which have been widely used for the development and standardization of a 3D epithelial cell culture (ex vivoproduced oral mucosa equivalent - EVPOME model), presented a full differentiated stratified epithelial equivalent (Feinberg et al., 2005; Izumi et al., 1999, 2004; Tobita et al., 2010). Porcine dermal matrices have also been demonstrated to produce a suitable organotypic epithelium (Basso et al., 2016a). The improved epithelial differentiation obtained for AlloDerm ${ }^{\circledR}$ and Strattice ${ }^{\circledR}$ may be related to the presence of a basement membrane, already demonstrated by Feinberg et al., (2005) - which is lacking on the collagen matrix - on the epidermal sides of these matrices.

The importance of fibroblasts for the phenotypes of dermal keratinocytes seeded on AlloDerm ${ }^{\circledR}$ matrices has already been demonstrated (Andriani et al., 2003). When both cells, fibroblasts and oral keratinocytes, were combined for the development of a full-thickness tissue, keratinocytes showed higher differentiation (stratification) in the presence of underlying fibroblasts. This study also evaluated the influence of the presence of gingival fibroblasts in the dermal matrices on the morphology and phenotype of oral keratinocytes; however, as demonstrated by Saito et al. (2014), mesenchymal cells did not migrate through the dermal matrix and were not homogeneously distributed, since only a few cells remained inside the matrix.

Also in the present investigation, the use of soluble collagen matrices provided a better distribution for these cells. Despite the ease of manipulation and processing of dermal matrices, results obtained in this study indicated that the collagen matrix model provided a more suitable method for a 3D gingival cell culture, where a more homogeneous distribution of fibroblasts with morphology similar to that of gingival tissue (Figs. 1c and 2c) was observed.

The normal gingival connective tissue is a highly cellular tissue that exhibits a homogeneous distribution of fibroblasts within the extracellular collagen-rich matrix (Bartold et al., 2000). Therefore, the comparison of the three matrices assessed in the present laboratory study demonstrated that a more similar pattern of the physiological condition was achieved for the collagen matrix, in which the fibroblasts showed cytoplasmic projections. The more uniform distribution observed for this collagen matrix might be due to the manipulation of fibroblasts with the collagen solution, allowing the cells to permeate this environment, remaining homogeneously distributed within the matrix during its contraction.

The evaluation of glucose consumption and cell proliferation also showed a distinct pattern for each model. For the collagen matrix model, associated with the distinct morphological characterization, the glucose consumption and proliferation of gingival fibroblasts were higher when these cells were seeded on the collagen matrix when compared with both dermal matrices (Fig. 3). However, for the full- thickness oral mucosa model, the AlloDerm ${ }^{\circledR}$ substrate provided higher values for cell proliferation. These results may be due to the presence of a basement membrane on the epidermal side of this matrix, resulting in higher adherence and increased differentiation of oral keratinocytes seeded on this matrix, as previously demonstrated (Basso et al., 2016a).

Also for the collagen model, gene expression of COLIA1 and hVEGF was also enhanced when compared with that on the AlloDerm ${ }^{\circledR}$ and Strattice ${ }^{\circledR}$ matrices (Fig. 4).

In contrast, gene expression of hEGF was higher for AlloDerm, which corresponds to the higher cell stratification observed by the morphological analysis (Fig. 5). However, these models showed decreased expression of hCOL-I and hVEGF when compared to collagen matrices, demonstrating that even in the presence of epidermal components, the mesenchymal cells showed improved phenotypic characterization when seeded within soluble collagen (Fig. 4).

Synthesis of these growth factors was also higher for constructs of dermal matrices, however, a more discrete difference was observed when compared to gene expression assay. This discrepancy may be due to the time-point of evaluation (Figs. 6 and 7).

Taken together, these results indicate that collagen matrix can be used for the development of 3D cellular gingival connective tissue, and that this model provides a more suitable method than a dermal matrix. However, further studies are needed to evaluate the phenotypes of gingival fibroblasts seeded on this model, as well as to assess the feasibility of application of different therapies and materials, to provide a better elucidation of responses of gingival connective tissue. In addition, this model may also be improved by the addition of other cell types, such as endothelial and immune cells, to the matrix, simulating other specific oral mucosa conditions.

\section{Acknowledgments}

The authors acknowledge the Fundação de Amparo à Pesquisa do Estado de São Paulo - FAPESP (grants: 2013/05879-0; PD: 2012/179478 and BEPE/PD: 2014/06057-7) for financial support and the LifeCell Corporation for donation of the matrices used in this in vitro study.

\section{Competing interests}

The authors have no competing interests to declare.

\section{References}

Andriani, F., Margulis, A., Lin, N., Griffey, S.Y., Garlick, A., 2003. Analysis of microenvironmental factors contributing to basement membrane assembly and normalized epidermal phenotype. J. Invest. Dermatol. 120, 923-931. http://dx.doi.org/10.1046/ j.1523-1747.2003.12235 x.

Bartold, P.M., Walsh, L.J., Narayanan, A.S., 2000. Molecular and cell biology of the gingiva. Periodontology 24, 28-55.

Basso, F.G., Hebling, J., Marcelo, C.L., de Souza Costa, C.A., Feinberg, S.E., 2016a. Development of an oral mucosa equivalent using a porcine dermal matrix. Br. J. Oral Maxillofac. Surg. 55, 308-311. http://dx.doi.org/10.1016/j.bjoms.2016.09.019.

Basso, F.G., Soares, D.G., Hebling, J., de Souza Costa, C.A., 2016b. Low-level lasertherapy in 3D cell culture model using gingival fibroblasts. Lasers Med. Sci. 31, 973-978. http://dx.doi.org/10.1007/s10103-016-1945-4.

Colley, H.E., Hearnden, V., Jones, A.V., Weinreb, P.H., Violette, S.M., Macneil, S., Thornhill, M.H., Murdoch, C., 2011. Development of tissue engineered models of oral 
dysplasia and early invasive oral squamous cell carcinoma. Br. J. Cancer 105, 1192-1582. http://dx.doi.org/10.1038/bjc.2011.403.

Donetti, E., Bedoni, M., Capone, P., Gualerzi, A., Tartaglia, G., Sforza, C., 2009. An in vitro model of human oral explants to study early effects of radiation mucositis. Eur. J. Oral Sci. 117, 169-174. http://dx.doi.org/10.1111/j.1600-0722.2009.00614.x.

Dongari-Bagtzoglou, A., Kashleva, H., 2006. Development of a highly reproducible threedimensional organotypic model of the oral mucosa. Nat. Protoc. 1, 2012-2018. http://dx.doi.org/10.1038/nprot.2006.323.

Feinberg, S.E., Aghaloo, T.L., Cunningham, L.L., 2005. Role of tissue engineering in oral and maxillofacial reconstruction: findings of the 2005 AAOMS research summit. J. Oral Maxillofac. Surg. 63, 1418-1425. http://dx.doi.org/10.1016/j.joms.2005.07. 004.

Hakkinen, L., Uitto, V., Larjava, H., 2000. Cell biology of gingival wound healing. Periodontology 24, 127-152.

Izumi, K., Takacs, G., Terashi, H., Feinberg, S.E., 1999. Ex vivo development of a composite human oral mucosal equivalent. J. Oral Maxillofac. Surg. 57, 571-577.

Izumi, K., Song, J., Feinberg, S.E., 2004. Development of a tissue-engineered human oral mucosa: from the bench to the bed side. Cells Tissues Organs 176, 134-152. http:// dx.doi.org/10.1159/000075034.

Klausner, M., Ayehunie, S., Breyfogle, B.A., Wertz, P.W., Bacca, L., Kubilus, J., 2007. Organotypic human oral tissue models for toxicological studies. Toxicol. in Vitro 21, 938-949. http://dx.doi.org/10.1016/j.tiv.2007.01.024.

Lambros, M.P., Parsa, C., Mulamalla, H., Orlando, R., Lau, B., Huang, Y., Pon, D., Chow, M., 2011. Identifying cell and molecular stress after radiation in a three-dimensional (3-D) model of oral mucositis. Biochem. Biophys. Res. Commun. 405, 102-106. http://dx.doi.org/10.1016/j.bbrc.2010.12.135.

Moharamzadeh, K., Brook, I.M., Van Noort, R., Scutt, A.M., Thornhill, M.H., 2007. Tissueengineered oral mucosa: a review of the scientific literature. J. Dent. Res. 86 , 115-124. http://dx.doi.org/10.1177/154405910708600203.

Moharamzadeh, K., Colley, H., Murdoch, C., Hearnden, V., Chai, W.L., Brook, I.M., Thornhill, M.H., Macneil, S., 2012. Tissue-engineered oral mucosa. J. Dent. Res. 91 642-650. http://dx.doi.org/10.1177/0022034511435702.

Rakhorst, H.A., Posthumus-Vans Slujis, S.J., Tra, W.M., Van Neck, J.W., Van Osch, G.J., Hovius, S.E., El Ghalbzouri, A., Hofer, S.O., 2006. Fibroblasts accelerate culturing of muclsa substitues. Tissue Eng. 12, 2321-2331.

Rodrigues, A.Z., Oliveira, P.T., Novaes Jr., A.B., Maia, L.P., Souza, S.L.S., Palioto, D.B., 2010. Evaluation of in vitro human gingival fibroblast seeding on acellular dermal matrix. Braz. Dent. J. 21, 179-185. http://dx.doi.org/10.1590/S010364402010000300001

Saito, T., Izumi, K., Shiomi, A., Uenoyama, H., Ohnuki, H., Kato, H., Terada, M., NozawaInoue, K., Kawano, Y., Takagi, R., Maeda, T., 2014. Zoledronic acid impairs reepithelialization through down-regulation of integrin $\alpha \mathrm{V} \beta 6$ and transforming growth factor beta signaling in a three-dimensional in vitro wound healing model. Int. J. Oral Maxillofac. Surg. 43, 373-380. http://dx.doi.org/10.1016/j.ijmo.2013.06.016.

Tobita, T., Izumi, K., Feinberg, S.E., 2010. Development of an in vitro model for radiationinduced effects on oral keratinocytes. Int. J. Oral Maxillofac. Surg. 39, 364-370. http://dx.doi.org/10.1016/j.ijom.2009.12.020.

Werner, S., Krieg, T., Smola, H., 2007. Keratinocyte-fibroblast interactions in wound healing. J. Investig. Dermatol. 127, 998-1008. http://dx.doi.org/10.1038/sj.jid. 5700786. 